\title{
Protocol Disposition Event
}

National Cancer Institute

\section{Source}

National Cancer Institute. Protocol Disposition Event. NCI Thesaurus. Code C74590.

The group of incidents that occur during a clinical trial and describe whether a subject completed the study epoch or the reason this event did not occur. The subject's disposition is often described for each phase of the study. 